Marianna Csörnyei, Eötvös University, Department of Analysis, Budapest, Múzeum Krt. 6-8, H-1088, Hungary, e-mail: csornyei@@cs.elte.hu

\title{
ON THE CHORD SET OF CONTINUOUS FUNCTIONS
}

It is well-known that for a given continuous function $f, f(0)=f(1)$ and for any natural number $n$ there exist $x_{n}, y_{n}=x_{n}+1 / n$ such that $f\left(x_{n}\right)=f\left(y_{n}\right)$. It is also known that if the graph of $f$ (or more generally a planar curve connecting the point 0 and 1 ) does not have a horizontal chord of length $a$ and $b$ respectively then there is no horizontal chord of length $a+b$ either (see [1]). It is almost immediate that the lengths of possible horizontal chords of $f$ form a closed set $F$ of the unit interval $[0,1]$, and according to the remark above its complement $G=[0,1] \backslash F$ is an additive set: $a \in G, b \in G, a+b \leq 1$ imply $a+b \in G$. C. Ryll-Nardzewski, Z. Romanowicz and M. Morayne raised the problem whether this additive property is not just necessary but also sufficient for a set to be the complement of the chord-set of some continuous function.

In this paper we answer their question affirmatively by proving the following theorem.

Theorem 1 Let $F \subset[0,1]$ be a closed set, and put $G=[0,1] \backslash F$. Suppose that $0,1 \in F$ and if $x, y \in G, x+y \leq 1$, then $x+y \in G$. Then there is a continuous function $f$ defined on $[0,1]$ such that $\{y-x: x, y \in[0,1], x<y$, $f(x)=f(y)\}=F$.

ProOF. Let

$$
[0,1]=\left(\bigcup_{n} G_{n}\right) \cup\left(\bigcup_{k} F_{k}\right) \cup(\partial F),
$$

where $G_{n}$ and $F_{k}$ are disjoint open intervals, $\cup_{n} G_{n}=G, \cup_{k} F_{k}=\operatorname{int} F$ and $\partial F$ is the boundary of $F$.

\footnotetext{
Key Words: continuous functions, chord length

Mathematical Reviews subject classification: Primary: 26A15

Received by the editors November 15,1996

* Research supported by the Hungarian National Foundation for Scientific Research, Grant No. T 019476.
} 
We define $f(x)=0$ if $x \in \partial F$ (in particular, $f(0)=f(1)=0), f(x)=$ $\operatorname{dist}\left(x,[0,1] \backslash F_{k}\right)$ if $x \in F_{k}$, and $f(x)=-\operatorname{dist}\left(x,[0,1] \backslash G_{n}\right)$ if $x \in G_{n}$. We claim that $f$ satisfies the requirements.

$f$ is clearly continuous (moreover, Lipschitz 1 ) on $[0,1]$. Let $x, y \in[0,1]$, $x<y, f(x)=f(y)$. We prove that $y-x \in F$. First we show that

$$
\text { if } x, y \in \partial F \text { and } x<y \text { then } y-x \in F \text {. }
$$

Indeed, if $x=0$ then $y-x=y \in \partial F \subset F$. If $0<x<1$ then let $x_{n} \rightarrow x, x_{n} \in$ $G$. Then $y-x_{n} \notin G$ (since $x_{n} \in G, y-x_{n} \in G$ would imply $y \in G$ ). Thus $y-x_{n} \in F$ and $y-x=\lim \left(y-x_{n}\right) \in F$, as $F$ is closed.

If $f(x)=f(y)=0$ then $x, y \in \partial F$ and thus $y-x \in F$ by $(*)$. Therefore we may assume that $f(x)=f(y) \neq 0$. Since $f>0$ in int $F f<0$ in $G$, and $f=0$ in $\partial F$, this implies that either $x, y \in \operatorname{int} F$ or $x, y \in G$.

Suppose first that $x, y \in F_{k}$ for some $k$. If $h=y-x \in G$ then $n \cdot h \in G$ for every $n \leq 1 / h$, which is impossible, since $h<\left|F_{k}\right|$ and thus $n \cdot h \in F_{k}$ for some $n$.

Next suppose that $x, y \in G_{n}=(u, v)$ for some $n$. Then $h=y-x<\left|G_{n}\right|$ and thus $v-h \in G_{n} \subset G$. If $h \in G$ then $v=h+(v-h) \in G$ which is impossible, since $v \in \partial F \subset F$.

Thus we may assume that $x \in(a, b)$ and $y \in(c, d)$, where $(a, b)$ and $(c, d)$ are different components of int $F$ or $G$. We shall consider the following cases separately.

(i) $x \leq(a+b) / 2, y \leq(c+d) / 2$ and $(a, b),(c, d) \subset G$;

(ii) $x \leq(a+b) / 2, y>(c+d) / 2$ and $(a, b),(c, d) \subset G$;

(iii) $x>(a+b) / 2, y>(c+d) / 2$ and $(a, b),(c, d) \subset G$;

(iv) $x>(a+b) / 2, y \leq(c+d) / 2$ and $(a, b),(c, d) \subset G$;

(v) $x \leq(a+b) / 2, y \leq(c+d) / 2$ and $(a, b),(c, d) \subset \operatorname{int} F$;

(vi) $x \leq(a+b) / 2, y>(c+d) / 2$ and $(a, b),(c, d) \subset \operatorname{int} F$;

(vii) $x>(a+b) / 2, y>(c+d) / 2$ and $(a, b),(c, d) \subset \operatorname{int} F$;

(viii) $x>(a+b) / 2, y \leq(c+d) / 2$ and $(a, b),(c, d) \subset \operatorname{int} F$.

If (i), (iii), (v) or (vii) holds then $y-x=c-a$ or $y-x=d-b$. Since $a, b, c, d \in \partial F$, this implies $y-x \in F$ by $(*)$. In the sequel we shall denote

$$
u=\left\{\begin{array}{ll}
a, & \text { if } x \leq(a+b) / 2, \\
b, & \text { if } x>(a+b) / 2
\end{array} ; \quad v=\left\{\begin{array}{ll}
c, & \text { if } x \leq(c+d) / 2, \\
d, & \text { if } x>(c+d) / 2
\end{array} .\right.\right.
$$


Let $\delta=|x-u|=|y-v|=|f(x)|=|f(y)|$.

Case (ii): $v \in F, u+2 \delta-\varepsilon \in G \Longrightarrow v-(u+2 \delta-\varepsilon) \in F ; v-(u+2 \delta-\varepsilon) \rightarrow$ $v-u-2 \delta=y-x \in F$. Case (iv): $v \in F, u-2 \delta+\varepsilon \in G \Longrightarrow v-(u-2 \delta+\varepsilon) \in F$; $v-(u-2 \delta+\varepsilon) \rightarrow v-u+2 \delta=y-x \in F$. Case (vi): Either $u=0$, and then $y-x=v-2 \delta \in F$; or $\exists a_{n} \rightarrow u, a_{n} \in G$, and then $v-2 \delta \in F$, $\Longrightarrow v-2 \delta-a_{n} \in F,(v-2 \delta)-a_{n} \rightarrow v-u-2 \delta=y-x \in F$. Case (viii) $\exists a_{n} \rightarrow u$, $a_{n} \in G, v+2 \delta \in F \Longrightarrow(v+2 \delta)-a_{n} \in F,(v+2 \delta)-a_{n} \rightarrow v+2 \delta-u=y-x \in F$.

This completes the first part of the proof $(f(x)=f(y) \Longrightarrow y-x \in F)$. Next we show that for every $d \in F$ there are $x, y \in[0,1]$ such that $x<y$, $y-x=d$ and $f(x)=f(y)$.

This is clear if $G=\emptyset$; so that we may assume $G \neq \emptyset$. If $(a, b)=G_{n}$ then for every $0 \leq c \leq b-a$ there are points $a \leq x \leq y \leq b$ such that $y-x=c$ and $f(x)=f(y)$. As we proved above, this implies $c \in F$ for every $c \in[0, b-a]$. Therefore $g=\inf G>0$. Then $(0, g)$ is (one of the) longest component of $\operatorname{int} F$, since there are elements of $G$ arbitrarily close to $g$, and the integer multiples of these elements also belong to $G$.

If $d \in \partial F$ then $x=0, y=d$ satisfy the requirements. Next let $d \in \operatorname{int} F$, $d \in(a, b)=F_{k}$. We have $f(d)-f(0)=f(d)>0$ and $f(b)-f(b-d)=$ $-f(b-d)<0$, since $b-d<b-a \leq g$ and $f$ is positive on $(0, g)$. Now $f$ is continuous, and thus $f(y)-f(y-d)$ must vanish for a $y \in[d, b]$, completing the proof.

\section{References}

[1] A. M. Yaglom and I. M. Yaglom, Non-elementary problems in elementary presentation, GITTL, Moscow, 1954, Problem 118, p. 60 (in Russian). 